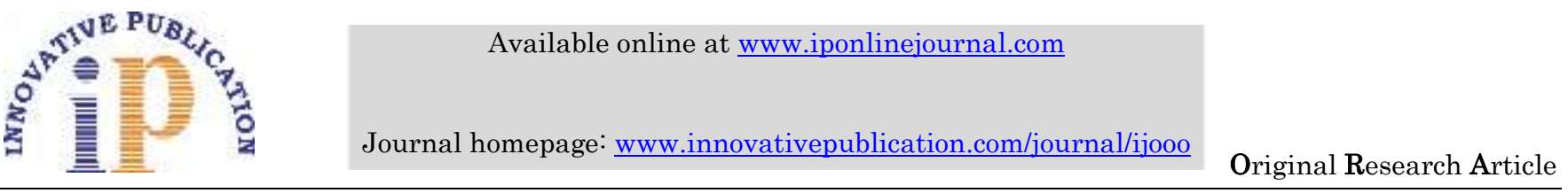

\title{
Pattern causes and management of eyelid and adnexal injuries in a tertiary hospital
}

\author{
Santanu Das ${ }^{1 *}$, Nayana Nagesh ${ }^{2}$, Raghuraj Hegde ${ }^{3}$ \\ ${ }^{\mathbf{1}} \mathrm{MS}$ Ophthalmology, ${ }^{\mathbf{2} J u n i o r}$ Resident, ${ }^{\mathbf{3}}$ Consultant, Oculoplasty and Ocular Oncology Services, ${ }^{\mathbf{1 - 3}}$ Dept. of Ophthalmology, Kempegowda \\ Institute of Medical Sciences, Bangalore, Karnataka, India
}

\begin{abstract}
Purpose: To describe the pattern, causes and management of eyelid and adnexal injuries in a tertiary care hospital.

Materials and Methods: A prospective study of 64 patients presenting to the emergency and outpatient department with eyelid and adnexal injuries from June 2017 to June 2019 with or without any globe injuries was carried out. Thorough ophthalmic evaluation was done for all. Wound was thoroughly washed with saline and broad-spectrum antibiotics were started for all patients. They were divided into only eyelid, canalicular and periocular injuries with or without globe injuries and orbital fractures. All the data were then analysed.

Results: Out of 64 patients 49 patients (76.5\%) with eyelid and adnexal injuries were due to road traffic accident (RTA).1was due to a bull horn injury. 7 were due to dog bite, 6 were due to self-fall and 1 was due to injury with cricket ball. 51(79.6\%) patients were male and 13 were female. Amongst the RTA cases 2 patients had avulsion injury. 1 patient had foreign body (glass pieces) inside the wound. 2 patients had associated ocular injuries. 26 patients had associated orbital fractures. 2 patients had associated ocular injuries. 27 patients had eyelid injuries. 40 patients had adnexal injuries whereas 7 patients had canalicular injuries. The most common complication encountered during the post-operative period was wound site scarring in 11 patients. They were treated with 5 fluoracil and triamcinolone injections. 1 patient had MRSA infection. Primary repair was the most common surgery performed. All the surgeries were performed within the first 72 hours of admission.

Conclusion: From our study we concluded that RTA is the most common cause and men were most commonly affected. Such injuries have varied presentations and management must be well planned and thought out to achieve good anatomical apposition as well as good cosmesis to avoid multiple surgeries.
\end{abstract}

Keywords: Adnexal injury, Birmingham Trauma Score, Canalicular injury, Eyelid injury.

\section{Introduction}

Eyelid and adnexal injuries can occur due to several causes and its incidence is on the rise owing to the increase in the number of road traffic accidents due to people not following rules and regulations properly while riding two wheelers or driving four wheelers. Eyelid and adnexal lacerations with associated globe injuries are a common emergency room challenge. A complete and thorough eye examination is essential before closing the wound to confirm the extent of injury, even if the wound appears simple. Each case of eyelid and adnexal injury are different and unique, and a thorough knowledge of the structural anatomy is needed to achieve good outcomes. The eyelids have excellent blood supply, which allows direct closure under tension and creation of large flaps without the threat of necrosis. ${ }^{1,2,3}$

3 important guidelines should be followed while performing closure:

1. Perform careful anatomic repairs.

2. Preserve the maximum possible amount of tissue.

3. Make liberal use of advancement flaps, traction sutures, and skin grafts.

Eyelid lacerations can be repaired within 72 hours of injury whereas canalicular injury can also be delayed (up to 48 hours), without great impact on the functional and aesthetic outcome. All tissue should be preserved, and even obviously necrotic tissue or detached tissue should be preserved until definitive closure is performed. Although eyelids and adnexa have excellent blood supply and can be repaired 72 hours after the initial trauma, the outcome also depends on the severity of the initial injury, first aid that has been provided, pre-existing eye conditions and the time within which the surgery was done. ${ }^{1,2,3,4}$

For injuries due to skid and fall from two-wheeler where the rider usually falls on one side of his face will present with the epidermis being scrapped off especially near the lateral canthal region needs a special mention as these injuries usually require local flaps or free skin graft for proper approximation and good cosmesis. This prospective study aims at highlighting:

1. Various modes and causes of eyelid and adnexal injuries in a tertiary care hospital.

2. Types and pattern of eyelid and adnexal injuries in a tertiary care hospital.

3. Management modalities of eyelid and adnexal injuries.

\section{Materials and Methods}

The following study adheres to the rules and regulations of the Declaration of Helsinki and has been approved the institutional ethics board. Before doing any procedure on our patients we have taken a written informed consent from

\footnotetext{
*Corresponding Author: Santanu Das, Dept. of Ophthalmology, Kempegowda Institute of Medical Sciences, Bangalore, Karnataka, India Email: shannn04@gmail.com

http://doi.org/10.18231/j.ijooo.2019.030
} 
all of them. Patients who didn't give their consent have not been included in the present study.

A prospective study of 64 patients presenting to the emergency and ophthalmology outpatient department with eyelid and adnexal injuries with or without associated globe injuries during June 2017 to June2019 were taken up for the study. They were divided mainly into

1. Eyelid

2. Canalicular

3. Other periocular soft tissue injuries

The injuries were further sub-classified as:

a. Without globe injury

b. With globe injury

c. Associated orbital and other facial fractures

Ocular injuries were classified as:

Zone 1: external (limited to bulbar conjunctiva, sclera and cornea)

Zone 2: anterior segment (including posterior lens capsule including pars plicata)

Zone 3: posterior segment (all internal structures posterior to the posterior lens capsule)

A thorough ophthalmic evaluation was done for each patient which included visual acuity, anterior segment evaluation under slit lamp, posterior segment evaluation with Indirect Ophthalmoscopy and B-scan. The anterior segment was evaluated for any corneal lacerations, foreign bodies, conjunctival tears, anterior chamber depth and reaction, lens subluxation, pupillary reaction to rule out relative afferent pupillary defect (RAPD) and any eyeball perforation or scleral lacerations.

CT-scan of orbits was done in cases of suspected orbital and facial fractures ( $1 \mathrm{~mm}$ cuts, bone windows, coronal, axial and sagittal views with $3 \mathrm{D}$ reconstruction). Injection Tetanus Toxoid $0.5 \mathrm{ml}$ intramuscularly was given to all the patients before proceeding with further treatment.

Preoperatively all the patients were started on broad spectrum intravenous antibiotics and steroids in cases of severe oedema. Regular dressing was done by irrigating the wound with normal saline, Povidone Iodine (5\%) and by removing dust and foreign particles and clearing slough so that the wound bed contains healthy granulation tissue.

All the primary repairs were done within the first 72 hours of admission except for 3 cases because one patient needed extensive dressing and clearing of slough because of pus discharge from the wound and one had high sugar levels. The third patient whose surgery was delayed presented to us with a left sided upper eyelid injury sutured outside and associated with preseptal cellulitis. The sutures were released, incision and drainage of the preseptal cellulitis was done and he was put-on broad-spectrum antibiotics. Regular dressing was done and after a week of treatment the eyelid laceration was sutured in layers. All the surgeries were done by a single surgeon.

For all the cases of dog bite at presentation the wound was first thoroughly cleaned with normal saline followed by infiltration with immunoglobulin all around the wound within the first 24 hours of the dog bite and primary repair was taken up 48 hours after immunoglobulin administration.

For all canalicular injuries primary repair with stenting was done. In cases of adnexal injuries with tissue loss where tissue apposition was difficult and was seen to cause traction local regional flaps were used to achieve proper approximation and cosmesis without causing any iatrogenic ectropion or entropion. If local regional flaps were not enough to cover the defect, then only free skin graft from the posterior auricular region was harvested and was placed over the defect.

14 Patients out of 26 with associated orbital fractures underwent orbital fracture repair through the transconjunctival route under general anaesthesia. 2 patients with globe injuries underwent globe repair first then their adnexal injuries were repaired.

Post operatively all the patients were treated with broad spectrum intravenous antibiotics and oral steroids for atleast 1 week to reduce the inflammation. Sutures were usually removed 10 days post-surgery whereas eyelid marginal sutures and graft sutures were kept in place for 2 weeks. Patients were followed up at 1 week, 1 month, 3 months and at 6 months post-surgery.

\section{Results}

In this prospective study of 64 patients the most common cause of trauma is road traffic accident as shown in Fig. 1. 49 patients $(76 \%)$ were involved in road traffic accidents whereas 8 patients (13\%) had animal inflicted wounds, $6(9 \%)$ had self-fall and cricket ball injury accounted for 1 patient (2\%).51(79.6\%) patients were male and $13(20.3 \%)$ were female. Male patients in the age group of 20 to 50 years were found to be most common.

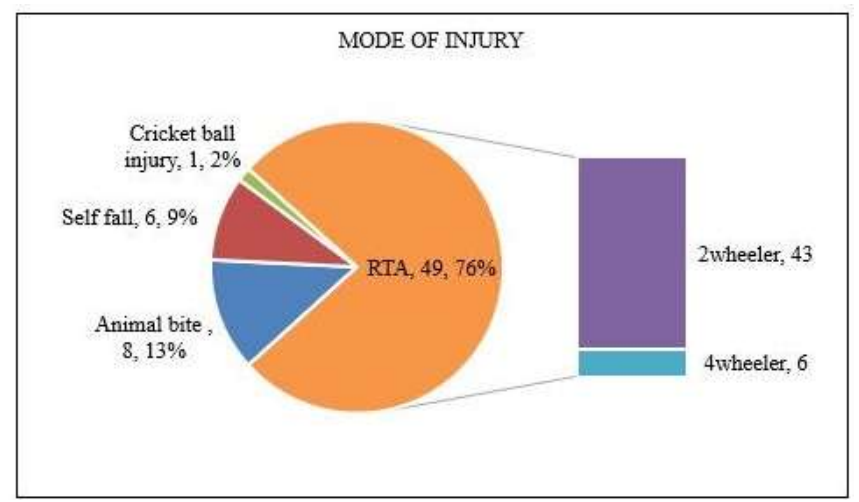

Fig. 1: (pie graph showing mode of injury)

Out of 49 cases of RTA, 43(81.4\%) were due to twowheeler accidents and6(18.5\%) were due to 4-wheeler accidents as shown in Fig. 1. Out of 43 two-wheeler accident patients, 7 patients $(16.2 \%)$ were pillion riders and $36(83.7 \%)$ were riders. Amongst 36 riders 34 (94.4\%) riders were not wearing helmet whereas 2 patients were wearing helmet. All the 7 patients who were pillion riders were 
female and were not wearing helmets. The types and causes

of RTA are depicted in figure 2 using a flowchart.

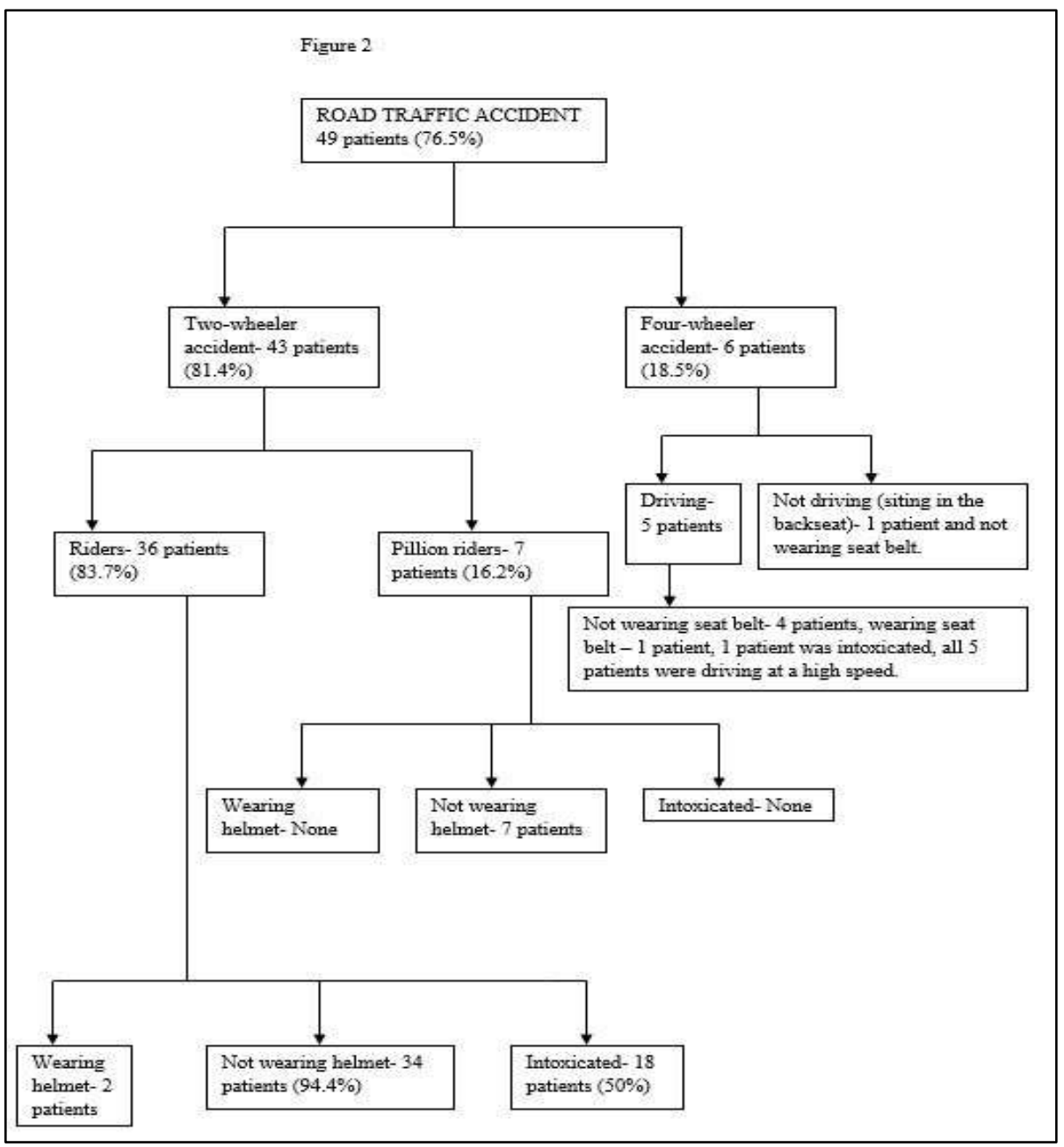

Fig. 2

Amongst the RTA cases 2 patients had avulsion injury. 1 patient had foreign body (glass pieces) inside the wound.2 patients had associated ocular injuries that is open globe injury. 26 patients had associated orbital fractures. 2 patients had associated ocular injuries. 27 patients had eyelid injuries. 40 patients had periocular and other soft tissue injuries which is most common. 7 patients had canalicular injuries. The varied modes of presentation of eyelid and adnexal injuries in our study is shown in Fig. 3.

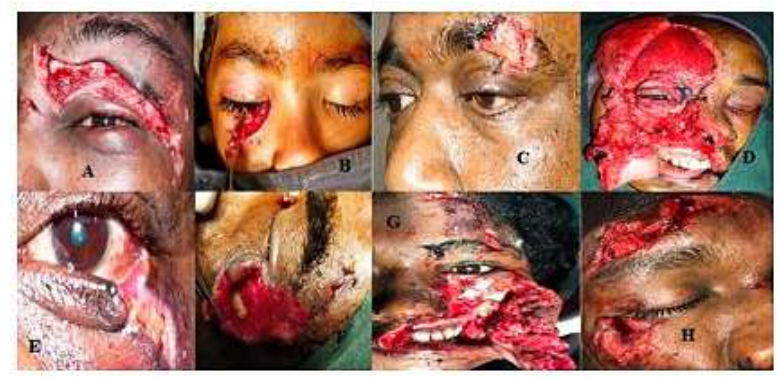

Fig. 3: (A) Left upper eyelid laceration with skin loss and a small laceration over the nasal bridge. (B) Right lower canalicular tear following dog bite. (C) Left central eyebrow defect with tissue loss following dog bite. (D) Right sided avulsion injury with upper and lower canalicular tear following RTA. (E) Left sided lower eyelid marginal defect. (F) Left sided lateral canthal injury with severe tissue loss. (G) Left sided avulsion injury after RTA. (H) Right sided lower eyelid laceration with forehead laceration due to RTA.

Amongst the 2 patients with associated open globe injury 1 patient had zone 1 injury whereas 1 patient had zone 3 injury. Following surgery there was no incidence of graft rejection or failure or necrosis of the graft. The donor site healed well in all the patients for whom a free skin graft was harvested. Some of the surgical outcomes we achieved are shown in Fig. 2. 


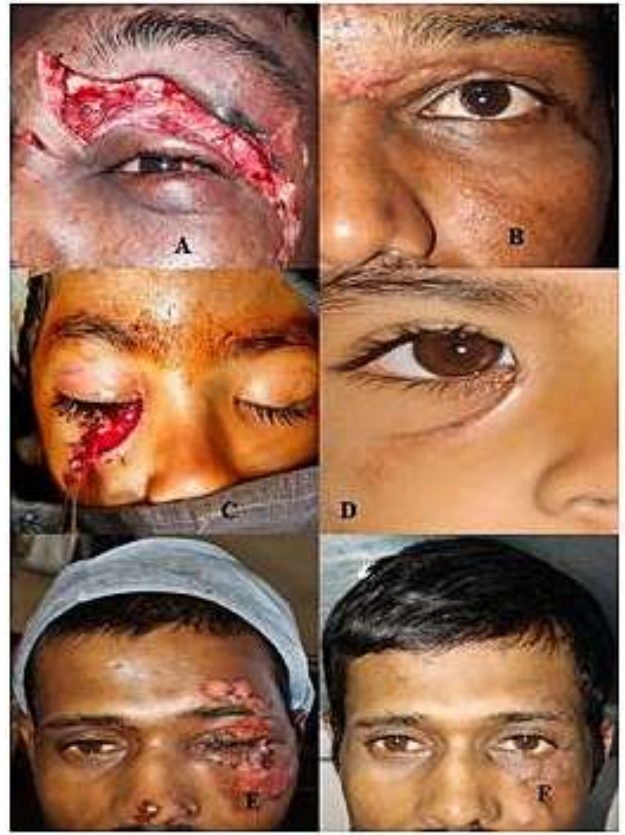

Fig. 4: (A) Shows left upper eyelid laceration with skin loss. (B) Shows the outcome at 6 months follow up. (C) Shows a right sided lower canalicular tear at presentation in a sixyear-old girl. (D) shows the outcome at 6months follow up with no anatomical and functional deficits. (E) Shows a left sided upper and lower eyelid defect with tissue loss whereas (F) Shows a good cosmetic and anatomical outcome at 6 months follow up.

One patient had MRSA infection. The most common complication encountered during the post-operative period was wound site scarring in 11 patients. These patients were treated with 5 Fluorouracil + Triamcinolone injections mixed in a ratio of 9:1 to soften up the scar. Injections were given each week around the scar tissue over a period of 2 to 3 months depending upon the response. 5 patients amongst these 11 patients needed scar release whereas one patient required scar release along with Z-Plasty surgery to obtain satisfactory cosmesis. One patient with an avulsion injury needed scar release with multiple Z-Plasty and Dermis fat grafting for achieving good cosmesis as well as volume augmentation as there was severe subcutaneous fat loss.

Primary apposition was the most common surgery done for 52 patients $(81.2 \%)$, canalicular repair with stenting was done for 7 patients (10.9\%), free skin graft was harvested from the post auricular area to reconstruct the wound in 4 patients $(6.2 \%)$ whereas a regional flap was required for 8 patients $(12.5 \%) .2$ patients with open globe injury underwent corneal laceration suturing with 10 'o Nylon sutures and scleral perforation repair with 6'o Vicryl sutures. The various surgical techniques used are shown in Fig. 5.

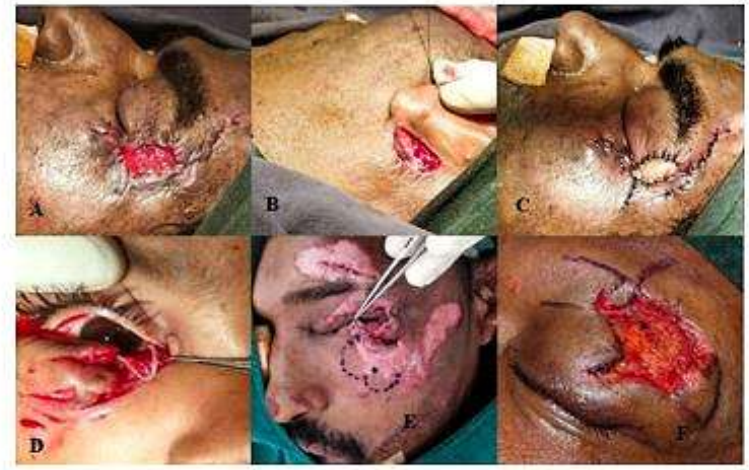

Fig. 5: (Various surgical techniques used). (A) Shows the defect left behind after primary apposition of the wound. (B) A free skin graft is being harvested from the posterior auricular area. (C) The free skin graft was used to cover the defect. (D) Canalicular repair is being done using a stent. (E) A bilobed flap being raised to cover the lower eyelid defect. (F) A pedicle island flap and an advancement flap being raised to reconstruct a central eyebrow defect.

\section{Discussion}

Injuries to the eyelids and ocular adnexa are very common, these injuries are often accompanied by intracranial or facial injuries, so stabilization of the patient and attention to the classic ABCs (airway, breathing, and circulation) of trauma care are crucial.

In our hospital-based study in Bangalore, India road traffic accident (RTA) was the most common mode of injury accounting for $76 \%$. Aparajita et al found penetrating injuries were the most common and RTA was the second most common mode of injury at $22.4 \%$ in their study. ${ }^{5}$ In the study conducted by Nirmalan et al in a rural south Indian population blunt trauma was found to be the most common cause. ${ }^{6}$ Usharani et al in their study found occupation related injuries were the most common mode of eye injury. Men in the age group of 20-30 years were most commonly involved while periocular and other soft tissue injuries were the most common presenting pattern in our study which is similar to the findings of Usharani et al. ${ }^{7}$ O.I. Okoye et al in their study found out that assault/combat, work related injuries and RTA constituted approximately $85.4 \%$ of the eye injuries admitted in their hospital in Nigeria. The most common causative agent being gunshot pellets, metal pieces, flying glass and wood splinters. ${ }^{8}$

Ilsar $\mathrm{M}$ et al found in their study that the most common mode of injury was chopping and gathering of wood. ${ }^{9}$ Vats et al found that blunt trauma was the most common mode of injury in an urban slum population in Delhi India which is similar to what Jain BS et al found in their study in north India. They found blunt trauma by stone especially amongst stone crushers was the commonest mode of blunt trauma. $^{10,11}$ This shows that the pattern of ocular and adnexal injuries depends on the geographic location, type of 
population and other factors like occupation also plays a role.

In our study male in the age group of 20-50 years, that is the working age group or the active age group people were most commonly affected which is like what other authors found. ${ }^{5-11}$

This leads to significant morbidity as well as loss of working years in severe cases.

Freda et al found in the paediatric age group the most common mode of injury was during playing with sharp objects in school and the mean age was 7.7 years. ${ }^{12}$ In our study all the paediatric cases we got had canalicular injuries and it was due to animal bites (dog bite).

In this study all the injuries were classified as per the Birmingham Eye Trauma Terminology as shown in Figure $6^{13}$

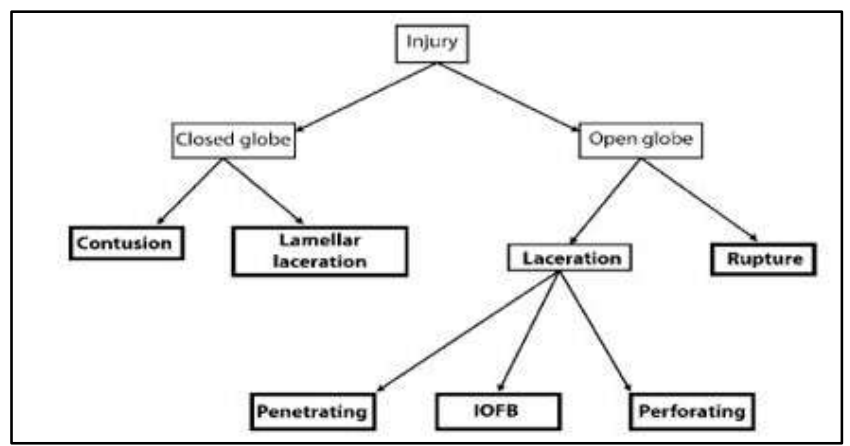

Fig. 6: (Birmingham Eye Trauma Terminology).

In this study $96.8 \%$ of the injuries were closed globe injuries which fell into the subcategory of lamellar laceration. Only 2 patients had open globe injury which fell into the subcategory of globe rupture. In our study globe rupture was found in 1 patient with open globe injury and 1 had corneal laceration. Globe rupture was also found to be the most common type of open globe injury in the study conducted by Saini et al. ${ }^{14}$ In our study closed globe injuries were most common which is similar to the results of the study conducted by Anette.K. Hoskin et al in Western Australia amongst athletes. ${ }^{15}$

The pre-operative, post-operative treatment protocol and the surgical techniques used during this study were like the protocol suggested by Nelson et al in his review article. At presentation for all our cases we cleaned the wound thoroughly, cleared the dust particles embedded in the wound and started intravenous broad-spectrum antibiotics. All the repairs were done within the first 72 hours and post operatively broad-spectrum antibiotics and regular wound dressing by maintaining asepsis was done. Primary apposition is the most commonly performed surgery in our study which has been described in literature as the best surgical procedure and flaps and grafts should be used liberally and carefully. ${ }^{2,4,16}$

As mentioned earlier scarring was the most common complication encountered by us, which we first treated with 5 Fluorouracil $(5 \mathrm{FU})+$ Triamcinolone $(\mathrm{TAC})$ injections and then if required a scar release or a Z-Plasty procedure was done. In the study conducted by Fitzpatrick in over 1000 patients he found injections of 5FU+TAC in the ratio of 9:1 produced more effective results and reduces the pain. He found it to be more effective than steroid injections alone. It has been stated by Ogawa Rand Wang XQ et al that 5-FU injections selectively blocks collagen synthesis as well as reduces the activity of fibroblasts which makes it very effective in treating postoperative scars. ${ }^{17,18}$

If primary closure was not possible, then only a combination of skin grafts, lid-sharing techniques, and flaps were used. The types of suture or canalicular stenting material are far less important than a meticulous primary closure which will ultimately restore the eyelid and adnexal anatomy. Finally, liberal use of traction sutures, pressure patches, and taping maximizes the cosmetic outcome.

\section{Conclusion}

Eyelid and adnexal injuries can have varied presentation and requires meticulous planning preoperatively to achieve best anatomical approximation as well as cosmesis. A multidisciplinary approach is required in most of the cases as most of these patients will have associated head injury and facial fractures. The patient should also be told about the need of multiple surgeries to achieve satisfactory cosmesis especially in cases of avulsion injury with distorted anatomy and adnexal injuries with extensive tissue loss. Lastly stricter rules while driving must be applied to prevent such complex adnexal injuries as they are not only detrimental for the cosmesis and vision but also it increases morbidity and causes loss of working years of life.

\section{Source of Funding}

None.

\section{Conflict of Interest}

None.

\section{References}

1. Grover AK, Bhatnagar A. Trauma to eyelids, canalicular lacerations and its management. JKSOS 1991;3(2):135-46.

2. Tomy RM. Management of eyelid lacerations. Kerala $J$ Ophthalmol 2018;30:222-7.

3. Christine C. Nelson, MD. Review of management of eyelid trauma. Aust New Zealand J Ophthalmol 1991;19(4).

4. Chang Eli L. M.D., Rubin, Peter A. D. M.D. Management of Complex Eyelid Lacerations. Int Ophthalmol Clin 2002;42:187-201.

5. Chaudhary A, Singh SP, Agasti M, Singh BK. Eye lid trauma and their management. Int J Ocular Oncol Oculoplasty 2016;2(4):240-3.

6. Nirmalan PK, Katz J, Tielsch JM et al. Ocular trauma in a rural South Indian population: The Aravind Comprehensive Eye Survey. Ophthalmol 2004;111:1778-81.

7. Laishram U, Yumnam CM, Gahlot A. Epidemiological profile of ocular trauma in a tertiary care facility in Imphal. $J$ Med Soc 2016; 30:162-5.

8. O. I. Okoye. Eye injury requiring hospitalisation in Enugu Nigeria: A one-year survey. Nigerian J Surg Res 2006;8(12):34-7. 
9. Ilsar M, Chirambo M and Belkin M. Ocular injuries in Malawi. Br J Ophthalmol 1982;66:145-8.

10. Vats S, Murthy GV, Chandra M, Epidemiological study of ocular trauma in an urban slum population in Delhi, India. Indian J Ophthalmol 2008;56:313-6.

11. Jain B.S. and Soni S.R. Ocular injuries: An analytical study in a teaching general hospital. IJO 1987;35:112-6.

12. Sii F, Barry JR, Abbott J. The UK Paediatric Ocular Trauma Study 2 (POTS2): demographics and mechanisms of injuries. Clin Ophthalmol 2018;12:105-11.

13. Kuhn F, Morris R, Witherspoon CD, Mester V. The Birmingham eye trauma terminology system (BETT). $J$ Fr Ophtalmol 2004;27:206-10.

14. Saini J S, Mukherjee A K, Dabral S M, Moraes O.A profile of penetrating eye injuries. IJO 1985;33:95-7.
15. Hoskin AK, E. Yardley AM, Kate Hanman K, Mackey. Sportsrelated eye and adnexal injuries in the Western Australian paediatric population. Acta Ophthalmol 2016;94:e407-e410.

16. Mutie D, Mwangi N. Managing eye injuries. Comm Eye Health J 2015;28(91):48-9.

17. Khan MA, Bashir MM, Khan FA. Intralesional triamcinolone alone and in combination with 5-fluorouracil for the treatment of Keloid and Hypertrophic scars. JPMA 2014;64:1003.

18. Gupta S, Sharma V K. Standard guidelines of care: Keloids and hypertrophic scars. Indian J Dermatol Venereol Leprol 2011;77:94-100.

How to cite this article: Das S, Nagesh N, Hegde R, Pattern causes and management of eyelid and adnexal injuries in a tertiary hospital. Int J Ocul Oncol Oculoplasty 2019;5(3):1127. 